\title{
Genetic Variability and $\mathrm{D}^{2}$ Analysis for Yield and Quality Traits in Tomato (Solanum lycopersicum L.)
}

\author{
Harsiddhi Limbani $^{1 *}$ and J. P. Makati \\ Department of Genetics and Plant Breeding, N. M. College of agriculture, Navsari \\ Agricultural University, Navsari, Gujarat, India \\ *Corresponding author
}

K e y w o r d s
Clusters, Diversity,
Genetic variability,
Quality, D ${ }^{2}$, Tomato
Article Info
Accepted:
$\begin{aligned} & 15 \text { April } 2020 \\ & \text { Available Online: } \\ & \text { 10 May } 2020\end{aligned}$

\section{A B S T R A C T}

Forty two genotypes of tomato including four check cultivar (GT-2, GT-6, JT-3 and Pusa Ruby) were planted in Randomized Block Design, during rabi 2018 and were assessed to know the nature and magnitude of variability and genetic divergence for twelve traits. The experimental results revealed a wide range of variability for all the traits under study. High heritability coupled with high genetic advance was observed for number of fruits per plant, plant height, fruit length, fruit girth, shelf life of fruits, tomato leaf curl virus incidence, average fruit weight, fruit borer damage and number of locules per fruit which offers the better scope for improvement through selection. Based on the Mahalanobis $\mathrm{D}^{2}$ statistics, forty two genotypes of tomato were grouped into three clusters. Maximum number of genotypes were accommodated in the cluster-I (40) followed by cluster-II (1) and clusterIII (1). Highest inter cluster distance of 273.83 was recorded between cluster I and III, hence, crossing between the genotypes of these cluster is expected to yield more heterotic hybrids. On the other hand, six genotypes viz., NTL-72, NTL-81, NTL-84, NTL-53, NTL65 and NTL-31 performed better for important traits under study. These genotypes need further testing to be released as a substitute of already existing tomato varieties or these can be crossed with diverse genotypes of other clusters for the development of superior hybrids in tomato.

\section{Introduction}

Tomato (Solanum lycopersicum $\mathrm{L}$., $2 \mathrm{n}=24$ ) is a member of solanaceae family, grown throughout the year in all over the world. It has wider adaptability, high yielding potential and multipurpose uses in fresh as well as processed food industries. Therefore, identification and development of new cultivars is important to improve production and productivity of tomato (Kumar et al., 2013a). Planning and execution of a breeding programme for the improvement of quantitative traits depends, to a great extent, upon magnitude of genetic variability (Kumar et al., 2013b). Genetic variability for yield and its component traits is essential in the base population for successful crop improvement (Allard, 1960). Tomato has a wide range of variability, which provides a tremendous scope for genetic improvement of its economic traits (Singh and Ramanujam, 
1981). An improvement in yield and quality of tomato is normally achieved by selecting the genotypes with desirable trait combinations existing in nature or by hybridization. The crop improvement also depends upon the extent to which desirable traits are heritable. Heritable variation can effectively be studied in conjunction with genetic advance. High heritability alone is not enough to make efficient selection in segregation, unless the information is accompanied for substantial amount of genetic advance (Johnson et al., 1955). Further, information on genetic diversity is used to identify the promising diverse genotypes, which may be used in further breeding programmes. Therefore, keeping in view the above facts in mind the present study has been conducted to obtain information on the extent of genetic variability and divergence among forty two genotypes of tomato and to assess their utility in developing heterotic combinations for commercial use.

\section{Materials and Methods}

The present experiment was conducted at NMCA college farm, Navsari Agricultural University, Navsari, during Rabi 2018-19. The soil of the experimental plot was black cotton soil with $\mathrm{pH}$ 7.4. Navsari is situated at $72^{\circ}$ 54' East longitude and $20^{\circ}$ 57' North latitude and at an altitude of $11.89 \mathrm{~m}$ above the mean sea level. This region falls under "South Gujarat Heavy Rainfall Zone, AES III". The climate of this zone is typically tropical and monsoonic. The average rainfall of the zone is about $1500 \mathrm{~mm}$ and is normally received by second fortnight of June and ceases by September end. Winter starts from November and ends by the middle of February. The experimental materials comprised of 42 genotypes of tomato (NTL-7, NTL-10, NTL-19, NTL-22, NTL-24, NTL25, NTL-26, NTL-28, NTL-31, NTL-38,
NTL-41, NTL-45, NTL-50, NTL-52, NTL57, NTL-58, NTL-63, NTL-64, NTL-65, NTL-66, NTL-68, NTL-71, NTL-72, NTL73, NTL-77, NTL-81, NTL-84, NTL-85, NTL-87, NTL-89, NTL-91, NTL-96, NTL99, NTL-105, NTL-17, NTL-21, NTL-48, NTL-53, GT-2, GT-6, JT-3, Pusa Ruby) collected from different indigenous and exotic sources. The experiment was laid out in Randomized Block Design at a spacing of 90 $\mathrm{cm} \times 45 \mathrm{~cm}$ in the plots with 3 replications. The standard agronomic practices were followed to maintain healthy crop stand. Except days to $50 \%$ flowering, fruit borer damage, tomato leaf curl virus damage, all other characters viz., plant height, branches per plant, no. of fruits per plant, fruit length, fruit girth, no. of locules per plant, shelf life of fruits, average fruit weight and fruit yield per plant observations were recorded on five randomly selected plants per plot excluding border plants. The mean values of data were subjected to the analysis of variance as per the procedure described by Panse and Sukhatme (1978). The genotypic and phenotypic coefficient of variation were calculated as per formulae given by Burton and De-Vane (1953). Heritability and genetic advance were calculated according to Allard (1960) and genetic gain was estimated as per the method given by Johnson et al., (1955). Multivariate analysis was done utilizing Mahalanobis $\mathrm{D}^{2}$ statistics and genotypes were grouped into different clusters following Tochers method as described by Rao (1952) and Mahalanobis (1936).

\section{Results and Discussion}

The results obtained from the present investigation as well as relevant discussion have been summarized as under.

The analysis of variance revealed significant genotypic differences for all twelve characters under study (Table 1). A wide range of 
variability was observed for different quantitative as well as qualitative traits indicating the scope for selection of suitable initial breeding material for further improvement. The mean performance of different genotypes as given in Table 2 revealed a wide range of variability for all the horticultural traits under study viz., Days to 50 per cent flowering (30.06\% to $46.26 \%$ ), plant height $(69.87 \mathrm{~cm}$ to $263.87 \mathrm{~cm})$, branches per plant (6.29 to 11.74), number of fruits per plant (15.34 to 138.32$)$, fruit length $(3.85 \mathrm{~cm}$ to $18.86 \mathrm{~cm})$, fruit girth $(0.69 \mathrm{~cm}$ to $4.85 \mathrm{~cm})$, number of locules per fruit (1.70 to 4.97), shelf life of fruits (2.15days to 8.08days), average fruit weight $(1.06 \mathrm{~g}$ to $86.54 \mathrm{~g}$ ), fruit yield per plant $(1.71 \mathrm{~kg}$ to $2.73 \mathrm{~kg}$ ), fruit borer damage $(1.89 \%$ to $26.45 \%)$ and tomato leaf curl virus incidence $(10.00 \%$ to $53.33 \%)$, which again revealed the existence of good deal of variability in the germplasm and offers the opportunity for improvement in yield and quality traits of tomato. The analysis of components of variance (Table 3) revealed that the genotypic variance followed the trend of phenotypic variance and was greater than environmental variance for all the characters except fruit yield per plant indicating that influence of environment on the expression of traits was lower or negligible. The genotypic variance was observed high in plant height, number of fruits per plant, average fruit weight, fruit borer damage and tomato leaf curl virus incidence, moderate in days to $50 \%$ flowering and Low in number of branches per plant, fruit length, fruit girth, number of locules per fruit, shelf life of tomato and yield per plant. Earlier workers like Dhanker and Dhanker (2006), Ghosh et al., (2010), Taisa et al., (2011), Madhurima and Amitava (2012), Ayush et al., (2012), Mohamed et al., (2012), Kumari and Sharma (2013), Patel et al., (2013), Khapte and Jansirani (2014), Rai et al., (2016), Das et al., (2017), Ligade et al., (2017) and Dutta et al., (2018) had also reported similar genotypic co-efficient of variation trends for different traits. The genotypic co-efficient of variation does not offer full scope to estimate the variations that are heritable and therefore, estimation of heritability becomes necessary. The estimates of heritability (broad sense) varied from $52.20 \%$ to $93.30 \%$ for different traits under study (Table 3). Further, genetic gain (expressed as per cent of population mean) was found low to high in nature and ranged from $8.42 \%$ to $113.20 \%$ for different traits (Table 3). In the present studies, high heritability with high genetic advance as percent of mean was recorded for number of fruits per plant, plant height, fruit length, fruit girth, shelf life of fruits, tomato leaf curl virus incidence, average fruit weight, fruit borer damage and number of locules per fruit. The result of the present study was in agreement with finding of Dhanker and Dhanker (2006), Ghosh et al., (2010), Madhurima and Amitava (2012), Ayush et al., (2012), Reddy et al., (2013), Hasan et al., 2016), Rai et al., (2016), Das et al., (2017) and Ligade et al., (2017), Thapa et al., (2018) and Dutta et al., (2018). It indicates the predominance of additive gene action and thus more reliable for selection. Moderate heritability with moderate genetic advance observed for days to $50 \%$ flowering and number of branches per plant indicated that these characters are under non-additive gene effects and selection for these characters will be less effective. Such traits are more under the influence of environment and do not respond to selection.

Genetic divergence: Information on genetic diversity is used to identify the promising diverse genotypes, which may be used in further breeding programmes. Based on the Mahalanobis $\mathrm{D}^{2}$ statistics, 42 genotypes of tomato were grouped into three clusters (Table 4). Maximum number of genotypes were accommodated in the cluster-I (40) followed by cluster-II (1) and cluster-III (1). 
Table.1 Analysis of variance for various traits in tomato

\begin{tabular}{|c|c|c|c|}
\hline Source of variation & Replication & Genotypes & Error \\
\hline Days to 50 per cent flowering & 25.95 & $52.79 * *$ & 9.96 \\
\hline Plant height (cm) & 197.17 & $3395.10 * *$ & 179.97 \\
\hline Branches per plant & 2.00 & $3.71 * *$ & 0.86 \\
\hline No. of fruits per plant & 63.69 & $1132.71 * *$ & 26.46 \\
\hline $\begin{array}{c}\text { Fruit girth } \\
\text { (cm) }\end{array}$ & 0.06 & $1.45 * *$ & 0.09 \\
\hline No. of locules per fruit & 0.01 & $1.97 * *$ & 0.24 \\
\hline Shelf life of fruits (Days) & 0.17 & $3.88 * *$ & 0.27 \\
\hline $\begin{array}{l}\text { Tomato leaf curl virus } \\
\text { incidence }(\%)\end{array}$ & 7.14 & $410.63 * *$ & 36.41 \\
\hline
\end{tabular}


Table.2 Mean values of genotypes for twelve characters of tomato (Solanum lycopersicum L.)

\begin{tabular}{|c|c|c|c|c|c|c|c|c|c|c|c|c|c|}
\hline $\begin{array}{l}\text { Sr. } \\
\text { No. }\end{array}$ & Genotypes & $\begin{array}{l}\text { Days to } \\
\mathbf{5 0} \% \\
\text { flowerin } \\
\text { g }\end{array}$ & $\begin{array}{l}\text { Plant } \\
\text { height } \\
\text { (cm) }\end{array}$ & $\begin{array}{l}\text { Branches } \\
\text { per plant }\end{array}$ & $\begin{array}{l}\text { No. of } \\
\text { fruits per } \\
\text { plant }\end{array}$ & $\begin{array}{l}\text { Fruit } \\
\text { length } \\
(\mathrm{cm})\end{array}$ & $\begin{array}{l}\text { Fruit } \\
\text { girth } \\
\text { (cm) }\end{array}$ & $\begin{array}{l}\text { No. of } \\
\text { locules } \\
\text { per fruit }\end{array}$ & $\begin{array}{l}\text { Shelf life } \\
\text { of fruits } \\
\text { (Days) }\end{array}$ & $\begin{array}{l}\text { Average } \\
\text { fruit } \\
\text { weight } \\
\text { (g) }\end{array}$ & $\begin{array}{l}\text { Fruit } \\
\text { yield per } \\
\text { plant(kg) }\end{array}$ & $\begin{array}{l}\text { Fruit } \\
\text { borer } \\
\text { damage } \\
(\%)\end{array}$ & $\begin{array}{l}\text { Tomato } \\
\text { leaf curl } \\
\text { virus } \\
\text { incidence } \\
(\%)\end{array}$ \\
\hline 1 & NTL-7 & 38.06 & 75.80 & 6.73 & 31.01 & 16.35 & 4.17 & 4.37 & 6.18 & 55.91 & 2.20 & 20.24 & 13.33 \\
\hline 2 & NTL-10 & 42.06 & 112.80 & 8.10 & 33.01 & 13.53 & 3.15 & 2.70 & 5.25 & 38.45 & 2.43 & 23.74 & 30.00 \\
\hline 3 & NTL-19 & 33.73 & 111.74 & 8.18 & 37.67 & 14.08 & 4.05 & 2.83 & 5.78 & 50.80 & 2.59 & 22.00 & 26.67 \\
\hline 4 & NTL-22 & 36.40 & 122.07 & 8.66 & 45.34 & 17.24 & 3.33 & 4.90 & 6.38 & 66.59 & 1.76 & 23.21 & 36.67 \\
\hline 5 & NTL-24 & 44.06 & 74.60 & 9.26 & 45.34 & 12.96 & 4.01 & 2.83 & 5.63 & 41.11 & 1.83 & 15.34 & 36.67 \\
\hline 6 & NTL-25 & 30.73 & 92.81 & 7.52 & 31.34 & 14.31 & 3.82 & 3.17 & 6.85 & 47.52 & 2.43 & 26.31 & 36.67 \\
\hline 7 & NTL-26 & 35.73 & 106.94 & 7.63 & 30.34 & 14.49 & 3.71 & 2.83 & 5.88 & 49.31 & 2.65 & 14.55 & 26.67 \\
\hline 8 & NTL-28 & 34.39 & 100.54 & 8.47 & 26.01 & 14.00 & 3.45 & 2.83 & 5.55 & 43.91 & 1.74 & 14.19 & 33.33 \\
\hline 9 & NTL-31 & 30.39 & 91.60 & 8.96 & 20.34 & 14.14 & 3.55 & 3.30 & 6.21 & 43.61 & 2.13 & 13.41 & 43.33 \\
\hline 10 & NTL-38 & 32.40 & 110.21 & 8.47 & 15.34 & 12.77 & 4.11 & 2.50 & 5.88 & 42.66 & 1.73 & 25.89 & 36.67 \\
\hline 11 & NTL-41 & 36.06 & 119.74 & 6.45 & 26.01 & 16.78 & 3.88 & 3.63 & 5.98 & 65.47 & 1.73 & 17.34 & 36.67 \\
\hline 12 & NTL-45 & 43.40 & 96.21 & 7.41 & 28.67 & 14.12 & 3.25 & 3.10 & 5.95 & 41.47 & 2.10 & 16.52 & 13.33 \\
\hline 13 & NTL-50 & 36.06 & 123.07 & 8.16 & 26.34 & 14.29 & 3.95 & 3.50 & 6.42 & 47.39 & 1.76 & 26.39 & 16.67 \\
\hline 14 & NTL-52 & 35.06 & 79.54 & 7.70 & 32.01 & 15.77 & 3.69 & 3.63 & 5.47 & 59.25 & 2.06 & 20.45 & 10.00 \\
\hline 15 & NTL-57 & 38.40 & 114.74 & 6.29 & 23.34 & 16.71 & 3.77 & 4.17 & 6.21 & 60.07 & 2.17 & 16.63 & 36.67 \\
\hline 16 & NTL-58 & 43.06 & 84.81 & 8.81 & 25.34 & 15.91 & 3.48 & 3.70 & 5.14 & 55.77 & 2.26 & 24.59 & 23.33 \\
\hline 17 & NTL-63 & 34.40 & 93.41 & 8.59 & 43.01 & 14.47 & 3.28 & 3.83 & 4.11 & 44.13 & 1.81 & 24.23 & 20.00 \\
\hline 18 & NTL-64 & 41.40 & 77.94 & 7.67 & 54.33 & 7.57 & 2.63 & 2.56 & 2.15 & 10.81 & 2.13 & 7.51 & 13.33 \\
\hline 19 & NTL-65 & 38.40 & 69.87 & 7.86 & 26.01 & 16.21 & 3.29 & 4.17 & 6.65 & 56.38 & 2.42 & 23.56 & 46.67 \\
\hline 20 & NTL-66 & 37.40 & 77.21 & 6.57 & 39.34 & 15.03 & 3.45 & 3.50 & 6.87 & 51.21 & 2.70 & 20.41 & 26.67 \\
\hline 21 & NTL-68 & 40.73 & 86.00 & 8.39 & 32.68 & 11.98 & 3.59 & 2.50 & 4.81 & 34.47 & 2.40 & 17.03 & 53.33 \\
\hline 22 & NTL-71 & 41.73 & 134.67 & 7.33 & 30.34 & 15.00 & 3.67 & 3.64 & 5.14 & 50.72 & 2.02 & 20.15 & 36.67 \\
\hline 23 & NTL-72 & 40.06 & 72.94 & 6.60 & 33.34 & 16.93 & $4.85^{*}$ & 2.70 & 6.01 & 66.05 & 2.73 & 25.82 & 13.33 \\
\hline
\end{tabular}


Contd...

\begin{tabular}{|c|c|c|c|c|c|c|c|c|c|c|c|c|c|}
\hline $\begin{array}{l}\text { Sr. } \\
\text { No. }\end{array}$ & Genotypes & \begin{tabular}{|lr} 
Days & to \\
$\mathbf{5 0}$ & $\%$ \\
flowering
\end{tabular} & $\begin{array}{l}\text { Plant } \\
\text { height } \\
\text { (cm) }\end{array}$ & $\begin{array}{l}\text { Branches } \\
\text { per plant }\end{array}$ & $\begin{array}{l}\text { No. of } \\
\text { fruits per } \\
\text { plant }\end{array}$ & $\begin{array}{l}\text { Fruit } \\
\text { length } \\
\text { (cm) }\end{array}$ & $\begin{array}{l}\text { Fruit } \\
\text { girth } \\
\text { (cm) }\end{array}$ & $\begin{array}{l}\text { No. of } \\
\text { locules } \\
\text { per plant }\end{array}$ & $\begin{array}{l}\text { Shelf life } \\
\text { of fruits } \\
\text { (Days) }\end{array}$ & $\begin{array}{l}\text { Average } \\
\text { fruit } \\
\text { weight } \\
\text { (g) }\end{array}$ & $\begin{array}{l}\text { Fruit } \\
\text { yield per } \\
\text { plant } \\
(\mathrm{kg})\end{array}$ & $\begin{array}{l}\text { Fruit } \\
\text { borer } \\
\text { damage } \\
(\%)\end{array}$ & $\begin{array}{l}\text { Tomato } \\
\text { leaf curl } \\
\text { virus } \\
\text { incidence } \\
(\%)\end{array}$ \\
\hline 24 & NTL-73 & 37.06 & 137.87 & 8.30 & 27.67 & 14.33 & 3.22 & 3.70 & 4.80 & 44.24 & 2.14 & 23.40 & 20.00 \\
\hline 25 & NTL-77 & 31.39 & 82.07 & 8.08 & 22.34 & 12.51 & 2.83 & 2.90 & 3.80 & 31.28 & 2.16 & 23.82 & 23.33 \\
\hline 26 & NTL-81 & 33.06 & 172.14 & 11.74 & 74.01 & 7.00 & 1.72 & 2.03 & 2.81 & 6.44 & 1.71 & 5.48 & 40.00 \\
\hline 27 & NTL-84 & 44.06 & 263.87 & 10.49 & 138.32 & 3.85 & 0.69 & 1.70 & 3.82 & 1.06 & 2.22 & 1.89 & 46.67 \\
\hline 28 & NTL-85 & 46.26 & 114.87 & 7.74 & 31.01 & 16.35 & 3.17 & 4.77 & 6.21 & 53.93 & 2.29 & 14.52 & 13.33 \\
\hline 29 & NTL-87 & 36.40 & 125.07 & 8.11 & 31.01 & 14.84 & 3.44 & 2.83 & 7.46 & 52.04 & 2.47 & 23.75 & 23.33 \\
\hline 30 & NTL-89 & 32.06 & 137.27 & 8.74 & 27.01 & 15.63 & 3.36 & 3.44 & 6.14 & 51.52 & 2.03 & 16.08 & 13.33 \\
\hline 31 & NTL-91 & 30.73 & 93.94 & 7.67 & 23.01 & 16.97 & 3.20 & 4.44 & 5.49 & 57.97 & 2.58 & 22.00 & 26.67 \\
\hline 32 & NTL-96 & 35.40 & 130.54 & 6.82 & 25.34 & 13.51 & 3.82 & 2.57 & 6.67 & 44.61 & 2.45 & 20.66 & 33.33 \\
\hline 33 & NTL-99 & 35.06 & 105.07 & 9.59 & 18.01 & 17.15 & 4.51 & 3.03 & 6.63 & 86.55 & 1.80 & 25.15 & 30.00 \\
\hline 34 & NTL-105 & 38.73 & 115.14 & 9.82 & 24.68 & 14.07 & 3.53 & 2.10 & 6.65 & 44.50 & 2.21 & 10.42 & 43.33 \\
\hline 35 & NTL-17 & 39.06 & 112.80 & 7.22 & 33.68 & 16.42 & 3.71 & 2.97 & 6.37 & 57.49 & 2.52 & 22.97 & 40.00 \\
\hline 36 & NTL-21 & 42.06 & 115.07 & 8.17 & 36.67 & 15.87 & 3.90 & 2.03 & 6.57 & 61.20 & 2.22 & 26.45 & 40.00 \\
\hline 37 & NTL-48 & 35.40 & 91.74 & 7.74 & 23.01 & 13.67 & 4.03 & 2.03 & 5.77 & 42.37 & 2.18 & 18.04 & 43.33 \\
\hline 38 & NTL-53 & 33.06 & 115.27 & 6.92 & 22.34 & 14.58 & 3.91 & 2.90 & 8.08 & 54.84 & 2.17 & 22.97 & 53.33 \\
\hline 39 & GT-2 & 37.73 & 83.14 & 7.23 & 24.68 & 18.86 & 3.77 & 4.97 & 6.41 & 71.01 & 2.30 & 17.26 & 36.67 \\
\hline 40 & GT-6 & 34.07 & 77.40 & 6.33 & 34.68 & 16.81 & 3.96 & 3.37 & 5.47 & 62.81 & 2.70 & 22.59 & 40.00 \\
\hline 41 & JT-3 & 30.06 & 82.27 & 8.40 & 29.34 & 14.34 & 4.32 & 2.17 & 5.80 & 52.48 & 2.61 & 21.41 & 33.33 \\
\hline 42 & $\begin{array}{l}\text { Pusa } \\
\text { Ruby }\end{array}$ & 38.06 & 140.74 & 7.63 & 34.34 & 16.71 & 4.20 & 3.63 & 6.47 & 62.68 & 1.77 & 18.52 & 23.33 \\
\hline \multicolumn{2}{|c|}{ Mean } & 37.00 & 107.75 & 8.01 & 33.75 & 14.48 & 3.56 & 3.20 & 5.76 & 49.10 & 2.20 & 19.45 & 30.71 \\
\hline \multicolumn{2}{|c|}{ S.Em \pm} & 1.82 & 7.75 & 0.54 & 2.97 & 0.68 & 0.17 & 0.28 & 0.30 & 4.81 & 0.23 & 1.77 & 3.48 \\
\hline \multicolumn{2}{|c|}{ C.D at $5 \%$} & 5.13 & 21.79 & 1.51 & 8.36 & 1.92 & 0.48 & 0.79 & 0.85 & 13.53 & 0.66 & 4.99 & 9.80 \\
\hline \multicolumn{2}{|c|}{ C.V \% } & 8.53 & 12.45 & 11.62 & 15.24 & 8.16 & 8.38 & 15.25 & 9.10 & 16.97 & 18.43 & 15.79 & 19.65 \\
\hline
\end{tabular}


Table.3 Range, mean and components of variance for various traits in tomato

\begin{tabular}{|c|c|c|c|c|c|c|c|c|}
\hline Sr.No. & Characters & Range & Mean & GCV \% & PCV \% & $\begin{array}{l}\text { Heritability } \\
\text { (b.s.\%) }\end{array}$ & $\begin{array}{l}\text { Genetic } \\
\text { advance }\end{array}$ & $\begin{array}{l}\text { Genetic } \\
\text { advance } \\
\% \text { of } \\
\text { mean }\end{array}$ \\
\hline 1. & $\begin{array}{l}\text { Days to } 50 \text { per cent } \\
\text { flowering }\end{array}$ & $30.06-46.26$ & 37.00 & 10.21 & 13.31 & 58.90 & 5.97 & 16.15 \\
\hline 2. & Plant height (cm) & $69.87-263.87$ & 107.75 & 30.38 & 32.83 & 85.60 & 62.40 & 57.91 \\
\hline 3. & Branches per plant & $6.29-11.74$ & 8.01 & 12.15 & 16.82 & 52.20 & 1.45 & 18.09 \\
\hline 4. & $\begin{array}{l}\text { No. of fruits per } \\
\text { plant }\end{array}$ & $15.34-138.32$ & 33.75 & 56.89 & 58.90 & 93.30 & 38.21 & 113.20 \\
\hline 5. & Fruit length (cm) & $3.85-18.86$ & 14.48 & 18.83 & 20.52 & 84.20 & 5.15 & 35.58 \\
\hline 6. & Fruit girth (cm) & $0.69-4.85$ & 3.56 & 18.94 & 20.71 & 83.60 & 1.27 & 35.67 \\
\hline 7. & $\begin{array}{l}\text { No. of locules per } \\
\text { fruit }\end{array}$ & $1.70-4.97$ & 3.20 & 23.77 & 28.24 & 70.80 & 1.32 & 41.21 \\
\hline 8. & $\begin{array}{l}\text { Shelf life of fruits } \\
\text { (days) }\end{array}$ & $2.15-8.08$ & 5.76 & 19.03 & 21.09 & 81.40 & 2.04 & 35.37 \\
\hline 9. & $\begin{array}{l}\text { Average fruit weight } \\
\text { (g) }\end{array}$ & $1.06-86.55$ & 49.10 & 31.04 & 35.38 & 77.00 & 27.54 & 56.10 \\
\hline 10. & $\begin{array}{l}\text { Fruit yield per plant } \\
\text { (kg) }\end{array}$ & $1.71-2.73$ & 2.20 & 9.18 & 20.59 & 19.90 & 0.19 & 8.42 \\
\hline 11. & $\begin{array}{l}\text { Fruit borer damage } \\
(\%)\end{array}$ & $1.89-26.45$ & 19.45 & 28.28 & 32.39 & 76.20 & 9.89 & 50.86 \\
\hline 12. & $\begin{array}{l}\text { Tomato leaf curl } \\
\text { virus incidence }(\%)\end{array}$ & $10.00-53.33$ & 30.71 & 36.36 & 41.33 & 77.40 & 20.24 & 65.90 \\
\hline
\end{tabular}


Table.4 Distribution of 42 genotypes of tomato into three different clusters on the basis of Mahalanobis $\mathrm{D}^{2}$ statistics

\begin{tabular}{|c|c|c|}
\hline Clusters & $\begin{array}{c}\text { Number of } \\
\text { genotypes }\end{array}$ & Genotypes \\
\hline I & 40 & NTL-7, NTL-10, NTL-19, NTL-22, NTL-24, NTL-25, NTL-26, NTL- \\
& & 28, NTL-31, NTL-38, NTL-41, NTL-45, NTL-50, NTL-52, NTL-57, \\
& NTL-58, NTL-63, NTL-64, NTL-65, NTL-66, NTL-68, NTL-71, NTL- \\
& & 72, NTL-73, NTL-77, NTL-81, NTL-87, NTL-89, NTL-91, NTL-96, \\
& & NTL-99, NTL-105, NTL-17, NTL-21, NTL-48, NTL-53, GT-2, GT-6, \\
& & JT-3, Pusa Ruby \\
\hline II & 1 & NTL-81 \\
\hline
\end{tabular}

Table.5 Average inter and intra cluster $\left(\mathrm{D}^{2}\right)$ values for 42 genotypes of tomato

\begin{tabular}{|c|c|c|c|}
\hline Clusters & I & II & III \\
\hline I & & & 273.83 \\
\hline II & 19.45 & 91.77 & 81.68 \\
\hline III & & & 0.00 \\
\hline
\end{tabular}


Table.6 Cluster means for twelve characters in forty two tomato genotypes

\begin{tabular}{|c|c|c|c|c|c|c|c|c|c|c|c|c|}
\hline Clusters & $\begin{array}{l}\text { Days to } 50 \\
\text { per cent } \\
\text { flowering }\end{array}$ & $\begin{array}{l}\text { Plant } \\
\text { height } \\
\text { (cm) }\end{array}$ & $\begin{array}{l}\text { Branches } \\
\text { per plant }\end{array}$ & $\begin{array}{l}\text { Number } \\
\text { of fruits } \\
\text { per plant }\end{array}$ & $\begin{array}{l}\text { Fruit } \\
\text { length } \\
\text { (cm) }\end{array}$ & $\begin{array}{l}\text { Fruit } \\
\text { girth } \\
(\mathrm{cm})\end{array}$ & $\begin{array}{l}\text { Number } \\
\text { of locules } \\
\text { per fruit }\end{array}$ & $\begin{array}{l}\text { Shelf } \\
\text { life of } \\
\text { fruits } \\
\text { (days) }\end{array}$ & $\begin{array}{l}\text { Average } \\
\text { fruit } \\
\text { weight } \\
\text { (g) }\end{array}$ & $\begin{array}{l}\text { Fruit } \\
\text { yield } \\
\text { per } \\
\text { plant } \\
\text { (kg) }\end{array}$ & $\begin{array}{l}\text { Fruit } \\
\text { borer } \\
\text { damage } \\
(\%)\end{array}$ & $\begin{array}{l}\text { Tomato } \\
\text { leaf curl } \\
\text { virus } \\
\text { incidence } \\
(\%)\end{array}$ \\
\hline I & 36.92 & 102.24 & 7.86 & 30.13 & 14.93 & 3.68 & 3.27 & 5.88 & 51.36 & 2.21 & 20.24 & 30.08 \\
\hline II & 33.06 & 172.14 & 11.74 & 74.01 & 7.00 & 1.72 & 2.03 & 2.81 & 6.44 & 1.71 & 5.48 & 40.00 \\
\hline III & 44.06 & 263.87 & 10.49 & 138.32 & 3.85 & 0.69 & 1.70 & 3.82 & 1.06 & 2.22 & 1.89 & 46.67 \\
\hline
\end{tabular}


Among different twelve traits studied tomato leaf curl virus incidence, number of fruits per plant, shelf life of fruits, fruit borer damage, fruit girth and plant height contributed very much towards genetic divergence. Based on inter-cluster distance, clusters III and I followed by II and I had maximum intercluster distance. Therefore, it is concluded that the genotypes belonging to these clusters should be inter-crossed in order to generate more variability and to improve tomato. Cluster I revealed maximum mean value for fruit length, fruit girth, number of locules per fruit, shelf life of fruits, average fruit weight and fruit borer damage. Cluster II revealed maximum values for number of branches per plant, while cluster III revealed maximum value for days to $50 \%$ flowering, plant height, number of fruits per plant, fruit yield per plant and tomato leaf curl virus incidence. Similar findings have been reported by Jogi et al., (2008), Meena and Bahadur (2013), Dar et al., (2015), Kumar et al., (2016), Hossain et al., (2016) and Spaldon and Kumar (2017). Therefore, it can be concluded that the selection of parents for hybridization should not be based on geographical diversity only, but it should have a base of both geographical origin as well as genetic divergence (Table 5 and 6).

From the present investigation it can be concluded that six genotypes viz., NTL - 72, NTL - 84, NTL - 81, NTL - 53, NTL - 65 and NTL - 31 performed better for important traits. These genotypes need further testing to be released as a substitute of already existing tomato varieties or these can be crossed with other genotypes for the development of superior tomato hybrids.

\section{References}

Allard, W.,1960. Principles of plant breeding. John Willey and Sons. Inc. London, pp. 83-108.
Ayush A. F., Serhan M., Shareef A., Naseer and Kutma M. H. 2012. Study of genetic parameters and character interrelationship of yield and some yield components in tomato (Solanum lycopersicum L.). International Journal of Genetics 2: 29-33.

Burton, G.W. and De Vane, E.H. 1953. Estimating heritability in tall fescue (Festuca arundinacea) from replicated clonal material. Agronomy journal 45: 478-481.

Dar, R. A., Sharma, J. P. and Ahmad, M. 2015. Genetic diversity among some productive genotypes of tomato (Lycopersicon esculentum Mill.). African Journal of Biotechnology 14(22):1845-1853.

Das, B., Murmu, D.K., Ghimiray, T.S., and Karforma, J. 2017. Estimation of Genetic Variability and Character Association of Fruit Yield and Quality Traits in Tomato. International Journal of Pure and Applied Bioscience 6(1):1587-1595.

Dhankhar, S. K. and Dhankhar, B. S. 2006. Variability, heritability, correlation and path -coefficient studies in tomato. Haryana Journal of Horticultural Sciences 35 (1\&2):179-181.

Dutta, P., Hazari, S., Karak, C. and Talukdar, S. 2018. Study on genetic variability of different tomato (Solanum lycopersicum L.) cultivars grown under open field condition. International Journal of Chemical Studies 6(5):1706-1709.

Ghosh, K. P., Islam, A. K. M. A., Mian, M. A. K. and Hossain, M. M. 2010. Variability and character association in $\mathrm{F}_{2}$ segregating population of different commercial hybrids of tomato (Solanum lycopersicum L.). Journal of Applied Sciences and Environment Management 14(2): 91-95.

Hasan, M. M., Al Bari, M. A., and Hossain, M. A. 2016. Genetic Variability and 
Traits Association Analysis of Tomato (Lycopersicon esculentum L.) Genotypes for Yield and Quality Attributes. Universal Journal of Plant Science 4(3): 23-34.

Hossin, A. Y., Harun-Ur-Rashid, M., Parveen, S., Rahman, M. S., Akter, R., and Rahim, M. A. 2016. Evaluation of Breeding Potential of Tomato Germplasm using $\mathrm{D}^{2}$ analysis. Advances in Bioresearch, 7(4).

Johnson, H.W., Robinson, H.F. and Comstock, R.E. 1955. Estimates of genetic and environmental variability in soybean. Agronomy Journal 47 (7): 314-318.

Khapte P. S. and Jansirani P. P. 2014. Genetic variability and performance studies of tomato (Solanum lycopersicum L.) genotype for fruit quality and yield. Trends in Biosciences 7:1246-1248.

Kumar, D., Kumar, R., Kumar, S., Bhardwaj, M.L., Thakur, M.C., Kumar, R. Thakur, K., Dogra, B.S., Vikram, A., Thakur, A. and Kumar, P. 2013a. Genetic variability, correlation and path coefficient analysis in tomato. International Journal of Vegetable Science 19(4):313-323.

Kumar, P. A., Reddy, K. R., Reddy, R., Pandravada, S. and Saidaiah, P. 2016. Genetic Divergence Studies In Tomato Genotypes. The Bioscam 7: 2097-24.

Kumar, S., Kumar, D., Kumar, R., Thakur, K.S. and Dogra, B.S. 2013b. Estimation of genetic variability and divergence for fruit yield and quality traits in cucumber (Cucumis sativus L.) in North-Western Himalays.Universal Journal of Plant Science 1(2): 27-36.

Kumari, S. and Sharma, M.K. 2013. Genetic variability in tomato (Solanum lycopersicum L.). Journal of Vegetable Science 40(1): 83-86.

Ligade, P. P., Bahadur, V. and Gudadinni, P. 2017. Study on Genetic Variability,
Heritability, Genetic Advance in Tomato (Solanum lycopersicum L.). International Journal of Current Microbiology and Applied Science 6(11):1775-1783.

Madhurina, M. and Amitava, P. 2012. Studies on genetic variability and characters association of fruit quality parameters in tomato. HortFlora Research Spectrum 1(2):110-116.

Mahalanobis, P.C. 1936. On the generalized distance in statistics. Proceedings of National Academic Science (India) 2: 79-85.

Mohamed, S. M., Ali, E. E. and Mohamed, T. Y. 2012. Study of heritability and genetic variability among different plant and fruit characters of tomato (Solanum lycopersicum L.). International Journal of Scientific and Technological Research 1(2):55-58.

Meena, O. P. and Bahadur, V. 2013. Assessment of breeding potential of tomato (Lycopersicon esculentum Mill.) germplasm using $\mathrm{D}^{2}$ analysis. The Bioscan 8(4):1145-1148.

Panse, V. G. and Sukhatme, P. V. 1978. Statistical Methods for Agriculture Workers (Second edition), ICAR, New Delhi.

Patel, S. A., Kshirsagar, D. B., Attar, A. V., Bhalekar, M. N. 2013. Study on genetic variability, heritability and genetic advance in tomato. Intternational journal of plant science vol., 8(1):45-47.

Rai, A. K., Vikram, A. and Pandav, A. 2016. Genetics and plant breeding: Genetic variability studies in Tomato (Solanum lycopersicum $L$.) for yield and quality traits. International Journal of Agriculture, Environment \& Biotechnology 9(5):739.

Reddy, B. R., Begum, H., Sunil, N. and Reddy, M. T. 2013. Genetic divergence studies in exotic collections of tomato (Solanum lycopersicum L.). 
International Journal of Agricultural Science 9(2):588- 592

Rao, R. 1952. Advanced statistical methods in biometrical research. John Willey and sons Inc., New York, pp. 357-363.

Singh, S.P. and Ramanujam, S. 1981. Genetic divergence and hybrid performance in Cicer arietinum. Indian Journal of Genetics 41: 268-276.

Spaldon, S. and Kumar, S. 2017). Genetic Divergence Studies for Quantative and Quality Traits in Tomato (Solanum lycopersicum L.). International Journal of Environment, Agriculture and Biotechnology 2(3): 1227-1231.
Tasisa J, Belew D, Bantte $\mathrm{K}$ and Gebreselassie W. 2011. Variablity, heritability and genetic advance in tomato (Lycopersicon esculentum Mill.) genotypes in West Shoa Ethiopia. American Eurasian Journal of Agricultural and Environmental Science 11: 87-94.

Thapa Bandan, Srivastava K. and Bhandari Hemraj. 2018. Assessment Studies on Genetic Variability Traits in Different Lines of Tomato (Solanum lycopersicum L.) International Journal of Current Microbiology and Applied Science 7(9): 824-829.

\section{How to cite this article:}

Harsiddhi Limbani and Makati, J. P. 2020. Genetic Variability and $D^{2}$ Analysis for Yield and Quality Traits in Tomato (Solanum lycopersicum L.). Int.J.Curr.Microbiol.App.Sci. 9(05): 2163-2174. doi: https://doi.org/10.20546/ijcmas.2020.905.247 